\title{
A New Method for Spherical Object Detection and Its Application to Computer Aided Detection of Pulmonary Nodules in CT Images
}

\author{
Xiangwei Zhang ${ }^{1}$, Jonathan Stockel ${ }^{2}$, Matthias Wolf ${ }^{2}$, Pascal Cathier ${ }^{2}$, \\ Geoffrey McLennan ${ }^{1}$, Eric A. Hoffman ${ }^{1}$, and Milan Sonka ${ }^{1}$ \\ ${ }^{1}$ University of Iowa, Iowa City, IA, 52242, USA \\ ${ }^{2}$ Siemens Medical Solutions, Malvern, PA, 19355, USA
}

\begin{abstract}
A novel method called local shape controlled voting has been developed for spherical object detection in 3D voxel images. By introducing local shape properties into the voting procedure of normal overlap, the proposed method improves the capability of differentiating spherical objects from other structures, as the normal overlap technique only measures the 'density' of normal overlapping, while how the normals are distributed in $3 \mathrm{D}$ is not discovered. The proposed method was applied to computer aided detection of pulmonary nodules based on helical CT images. Experiments showed that this method attained a better performance compared to the original normal overlap technique.
\end{abstract}

\section{Introduction}

Lung cancer is one of the most lethal kinds of cancer worldwide. Its cure depends critically on disease detection in the early stages. Computed tomography (CT) is an important tool for early detection of nodules, but interpreting the large amount of thoracic CT images is a very challenging task for radiologists. Computer-aided detection (CAD) is considered as a promising tool to aid the radiologist in lung nodule $\mathrm{CT}$ interpretation.

Many techniques of nodule detection have been developed based on thoracic CT images. Giger [1] proposed multiple gray-level thresholding and a rule based method. Armato [2] introduced some 3D geometric features and a linear discriminant analysis (LDA) classifier. Kanazawa 3. used fuzzy clustering method and a rule-based method. Lee [4] used pherical/half-circle templates to detect in-field/pleural nodules respectively. Surface normal overlap method [5] was proposed to capture the concentration of surface normals. Surface curvature/local shape feature was also utilized for detecting lung nodules [6], 7].

In this work, we developed a new method called local shape controlled voting for spherical object detection in 3D voxel images. This new scheme integrates surface curvature feature into the voting procedure of normal overlap 5 . Specifically, we applied the proposed method to lung nodule CAD. The overall scheme of the CAD system is described in Sect. 2, the method is described in Sect. 3 . then the experimental results are presented in Sect. 4. and the conclusion is given in Sect. 5.

N. Ayache, S. Ourselin, A. Maeder (Eds.): MICCAI 2007, Part I, LNCS 4791, pp. 842 849, 2007.

(C) Springer-Verlag Berlin Heidelberg 2007 


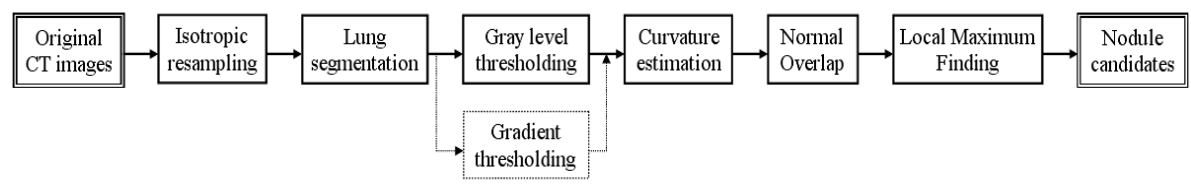

Fig. 1. Schematic view of the CAD for nodule detection
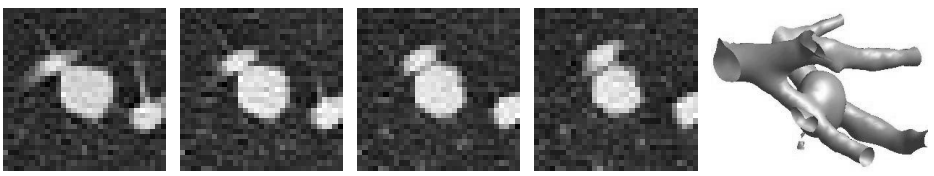

Fig. 2. A nodule attached to vessels. The left four images are neighboring slices in axial view, the most right one is an iso-surface rendering image.

\section{Overall Scheme}

The proposed method for pulmonary nodule detection is outlined in Fig. 1 . First, the original CT data are resampled to isotropic using tri-linear interpolation. Then the lung is extracted. Next, thresholding gives us high intensity regions. For each voxel near the boundary, shape index/curvedness features can be calculated using iso-surface curvatures. These features are used to control the voting procedure of normal overlap. For each voxel, the resulted value is a confidence score indicating the possibility of being the center of a nodule. The nodule candidate positions are obtained using local maximum criteria and thresholding.

\section{Methods}

\subsection{Basic Shape of Pulmonary Nodules}

In automated detection of nodules, dealing with non-isolated nodules (attaching to other structures) are major difficulties. Most methods first obtain suspected nodule areas (SNAs) based on intensity, then these SNAs are classified using associated features. Problem arises if a non-isolated nodule is segmented as part of normal structures, so it is difficult to be located afterwards.

Basic observations show that a nodule usually takes a sphere-like shape (or partly), while vessels have tubular structures. See, for example, Fig. 2, Usually this shape property was used as a feature of a segmented object, i.e., a SNA. In order to prevent from treating a non-isolated nodule as a part of attached structures, we need some local shape feature to distinguish between a non-isolated nodule and the contact normal anatomical structures.

\subsection{Surface Normal Overlap}

In order to detect sphere-like structures, a Hough transform type method, called surface normal overlap method [5] is developed to capture the concentration of 


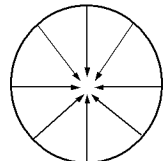

(a)

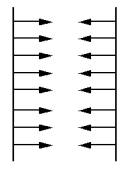

(b)

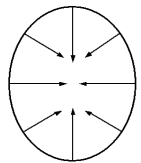

(c)

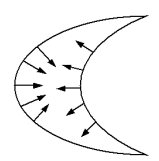

(d)

Fig. 3. Illustration of $2 \mathrm{D}$ boundary normal overlap

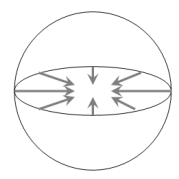

(a)

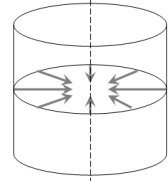

(b)

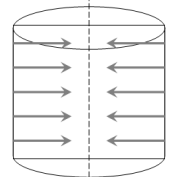

(c)

Fig. 4. Illustration of 3D boundary normal overlap

normals, illustrated in Fig. 3. The normals on the object boundary is assumed going inside. For a circle, all normals will meet at the center; for a strap like shape, a normal vector will only meet one normal from the other side; for an ellipse, the normals are less concentrated than a circle; for a complicated shape, the normals on the convex part will concentrate, while the normals on the concave part will diversify. 3D case is shown in Fig. 4. A sphere has the most concentrated normals at the center; any great circle will have a distribution same as a circle in 2D. For a cylinder, the cross section perpendicular to the axis is a circle; the cross section passing the axis is a strap like shape.

Assuming unit line/surface density on the boundary, then for a circle with radius $r$, the corresponding 'density' at the center is the circumference $2 \pi r$; for a strap-type object, the 'density' will be no more than two. For a sphere with radius $r$, the 'density' at the center will be the surface area of sphere $4 \pi r^{2}$; for a cylinder with directrix being a circle with radius $r$, the 'density' at the center line will be $2 \pi r$.

Thus, gradient directions can be used to detect circular/spherical structures in digital images. For pixels satisfying some boundary conditions, a tracking operation is performed in the gradient direction. This can be considered as a voting: every object pixel is a candidate; every boundary pixel is an individual voter who selects the favorite candidates (any object pixel on the gradient direction). The positions around the center of circular/spherical structures may have a high value after the voting procedure.

In a specific application, usually the maximum size of the object of interest is known in advance, thus the maximum voting distance can be set. Another idea is that the voting does not always need to reach the maximum distance, as the nodule candidate position should be inside the high intensity objects. 


\subsection{Air/Non-air Segmentation Using Optimal Thresholding}

Optimal thresholding [8] is used to automatically determine a threshold for segmentation between the background (parenchyma) and objects (nodules, vessels, and so on). Let $T^{t}$ be the threshold at iteration step $t$, and $\mu_{b}^{t}$ and $\mu_{o}^{t}$ be the mean intensity of background and objects. $\mu_{b}^{t}$ and $\mu_{o}^{t}$ are obtained by applying $T^{t}$ to the image. Then for the next step $t+1$, the threshold is updated by $T^{t+1}=\left(\mu_{b}^{t}+\mu_{o}^{t}\right) / 2$. This updating is iterated until the threshold does not change. The initial threshold $T^{0}$ is chosen as the mean value of the whole image.

\subsection{Local Maximum Finding}

After the voting, the pixels around the center of a circle/sphere will possibly have a higher value not only than their neighbors, but also higher than other non-circle or non-sphere structures. So the positions of nodule candidates can be generated by a combination of thresholding and local maximum criterion. In this work, the neighborhood used to check the local maximum criterion for a pixel is chosen as $3 \times 3 \times 3$. The rule to decide a local maximum is that a pixel is not less than any neighbor.

\subsection{Local Shape Features}

According to Sect. 3.2, the ratio of maximum 'density' of normals between a sphere with radius $r_{1}$ and a cylinder with radius $r_{2}$ is $4 \pi r_{1}^{2}$ to $2 \pi r_{2}$. With same radius $r$ (assuming that $r \geq 1$ for our applications), the sphere will have a higher density than the cylinder. But if $r_{2}$ is much larger than $r_{1}$, the cylinder will possibly give a higher response. This shows that the normal overlap method fails to distinguish between a small size sphere and a much larger size cylinder.

Practically, a nodule is usually not perfectly spherical; and vessels may have distortions resulting more concentrated normals; in addition, non-isolated nodules may only show spherical-like structure partly. All these factors can destroy the possible difference between nodules and non-nodules. To deal with these problems, we introduces local shape information to improve the original normal overlap technique [5].

A local surface shape can be completely described by its two principal curvatures, i.e., the maximal and the minimal curvatures $k_{1}, k_{2}$, or equivalently by the Gaussian curvature $K$ and the mean curvature $H$, see the $H K$ segmentation 9]. Neither the $H K$ curvatures pair nor the two principal curvatures pair capture the intuitive notion of "local shape" very well. In both schemes, two parameters are needed to "tell" the local shape. Koenderink 10 proposed two measures "shape index" $S$ and "curvedness" $C$. The shape index is scale-invariant and captures the intuitive notion of "local shape", whereas the curvedness specifies the amount of curvature.

$$
S=\frac{2}{\pi} \cdot \arctan \frac{k_{1}+k_{2}}{k_{1}-k_{2}}, \quad C=\sqrt{\frac{k_{1}^{2}+k_{2}^{2}}{2}}, \quad \text { for } k_{1} \geq k_{2}
$$


The $S$ and $C$ decouple the shape and the magnitude of the curvatures. This is done by transforming a $k_{1}, k_{2}$ Cartesian coordinate description of a local shape into a polar coordinate system description. Every distinct shape, except for the plane, corresponds to a unique value of $S . S=1$ indicates a cap, $S=-1$ describes a cup; $S=0.5$ (ridge) and $S=-0.5$ (rut) correspond to parabolic points (cylindrical shapes). For $0<S<0.5$, the local shape is a saddle (hyperbolic) ridge. For $-0.5<S<0$, saddle ruts are obtained. And symmetrical saddles have $S=0$. The range $-1<S<-0.5$ represents the concavities, and the range $0.5<S<1$ represents the convexities. A plane has a zero value $C$ and indeterminate $S$.

By assuming the surface of interest to be a level surface (iso-intensity surface) locally, curvatures can be computed from up to the second order partial derivatives (Gaussian smoothing with window size $7 \times 7 \times 7$ is used in the partial derivatives estimation in this work) of the image function [1]:

$$
\begin{aligned}
K & =\frac{1}{\left(f_{x}^{2}+f_{y}^{2}+f_{z}^{2}\right)^{2}}\left\{f_{x}^{2}\left(f_{y y} f_{z z}-f_{y z}^{2}\right)+2 f_{y} f_{z}\left(f_{x y} f_{x z}-f_{x x} f_{y z}\right)\right. \\
& +f_{y}^{2}\left(f_{x x} f_{z z}-f_{x z}^{2}\right)+2 f_{x} f_{z}\left(f_{x y} f_{y z}-f_{x z} f_{y y}\right) \\
& \left.+f_{z}^{2}\left(f_{x x} f_{y y}-f_{x y}^{2}\right)+2 f_{x} f_{y}\left(f_{x z} f_{y z}-f_{x y} f_{z z}\right)\right\} \\
H & =\frac{-1}{2\left(f_{x}^{2}+f_{y}^{2}+f_{z}^{2}\right)^{3 / 2}}\left\{\left(f_{y}^{2}+f_{z}^{2}\right) f_{x x}+\left(f_{x}^{2}+f_{z}^{2}\right) f_{y y}+\left(f_{x}^{2}+f_{y}^{2}\right) f_{z z}\right. \\
& \left.-2 f_{x} f_{y} f_{x y}-2 f_{x} f_{z} f_{x z}-2 f_{y} f_{z} f_{y z}\right\}
\end{aligned}
$$

The shape index of each voxel on the boundary of nodules tends to be around 1 due to the sphere-like local shape. And tubular vessels have the shape index value of 0.5 . For the airway walls, the voxels near the outer surface show the shape index value of 0.5 , while the voxels near the inner surface take the value -0.5 . This analysis shows that the shape index value can be used to differentiate the boundaries between of a nodule and vessels/airway walls. But this differentiation tends to be not very reliable due to noise, as it only depends on operation in a small neighborhood. What is needed is to synthesize these local information in a global way to give a more robust shape feature. Normal overlap technique will serve as the global level information processing tool in this work.

\subsection{Local Shape Controlled Voting}

In the original normal overlap scheme, every boundary point stimulates a tracking procedure. Such a system treats all voter equally. This rule is modified in the proposed local shape controlled voting: only boundary points satisfying some local shape conditions are allowed to vote, and the voting weights also depend on the local shape feature.

To exclude tubular structures (vessels, airways), a threshold $S_{t}$ can be set so that boundary points with $S \leq S_{t}$ are prohibited from the voting. Experiments 


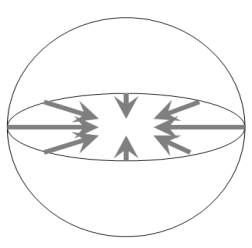

(a)

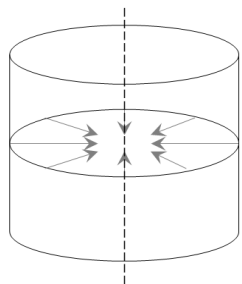

(b)

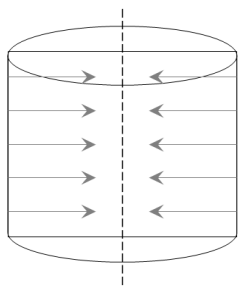

(c)

Fig. 5. Illustration of 3D local shape controlled voting.

show that $S_{t} \in[0.5,0.6]$ is a good choice for nodule detection. For boundary points with $S>S_{t}$, a voting procedure is started along the gradient direction, with the voting weight being $b\left(S-S_{t}\right)$, in order to enhance sphere-like local structures. Here $b$ is a positive coefficient. This local shape controlled voting is illustrated in Fig. 5. the thicker arrows represent larger weights; the thinner arrows indicate smaller weights.

It is also possible to use curvedness $C$ to drive the voting. If a boundary point has a very small curvedness, it is probably located at the slowly changing pleural surface, rather than on the boundary of a nodule with very limited size. So an upper bound threshold $C_{u}$ can be set to prohibit the voting. It is also possible to set a lower bound threshold $C_{l}$ in order to reduce the response from too tiny structures or noise.

Compared to the normal overlap method, it seems that we always pay extra cost for calculating local shape, but this is not true, because there are many boundary points not allowed to vote due to non-qualification. In the applications of nodule detection in CT images, most of the anatomical structures will either be tubular, such as vessels and airways, or be a slowly changing surface, such as pleural surface and lobe fissure. This situation is very suitable for the proposed method, as boundary points on these objects will not stimulate a voting. And experiments also showed the efficiency of the scheme, see Sect. 4.

\section{Experimental Methods and Results}

\subsection{Database}

The database consisted of 42 clinical high resolution helical thoracic CT cases. In this work, the independent standard is the nodule center positions created by human experts, i.e., the $x, y, z$ coordinates. Furthermore, the maximum 2D diameters of these nodules were measured manually.

\subsection{Validation}

A nodule is said to be detected by the CAD system if there is a position given by the CAD that is located closer than the size (the $2 \mathrm{D}$ maximum diameter 


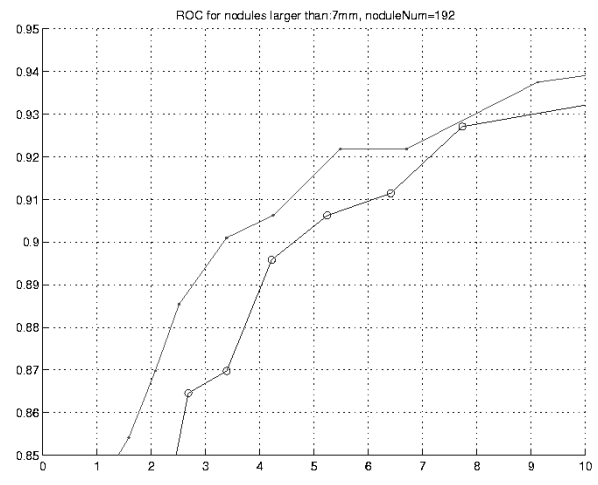

(a)

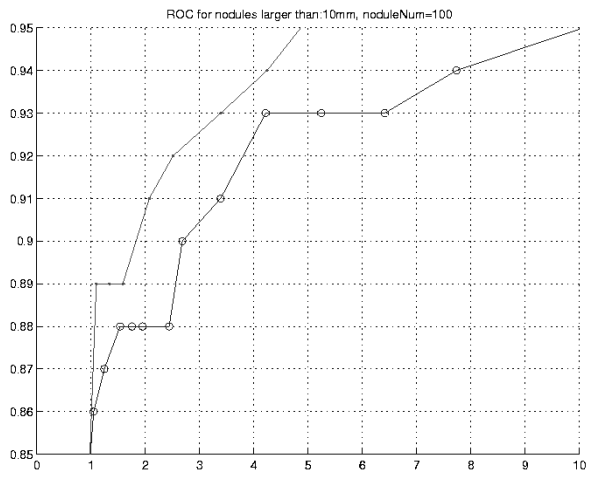

(b)

Fig. 6. Comparison of FROCs for the proposed method (.) and the original normal overlap method (o). The horizontal axis indicates the false positive number per case, and the vertical axis indicates the nodule detection sensitivity. (a) FROCs for nodule as small as $7 \mathrm{~mm}$; (b) FROCs for nodule larger than $10 \mathrm{~mm}$.

created manually, as described above) of the nodule. If there are more than one such positions for a single true nodule, only the position nearest to the true nodule is treated as the detected true nodule position, all the other positions are considered as FPs.

The value of the local maximum positions obtained from local shape controlled voting can be considered as the confidence score of being the center of nodules. FROC curves can be obtained accordingly, shown in Fig 6. It showed that the proposed local shape controlled voting method attained a better overall performance than the original surface normal overlap method. Here only nodules larger than $7 \mathrm{~mm}$ are evaluated, as these nodules are of significant clinical importance.

The efficiency of the proposed method is also investigated. The average processing time of local shape controlled voting (excluding isotropic resampling, lung segmentation) for each case is around 24.2 seconds, compared to the average time 30.1 seconds for surface normal overlap. The reason for this is that the average number of qualified voter decreased dramatically, only $12.9 \%$ of the boundary points in the direct normal overlap procedure was allowed to stimulate a tracking under the local shape standards.

\section{Conclusions}

A novel method called local shape controlled voting was proposed for spherical object detection in 3D voxel images. This scheme improved surface normal overlap method proposed for detection of spherical objects by integrating differential characteristics into the global tracking procedure of normal overlap. The proposed method has been applied to computer aided detection of pulmonary 
nodules based on helical CT images. Validation on 42 high resolution clinical cases showed that this method attains a better performance compared to the original normal overlap technique. Additionally, the time efficiency is also improved.

\section{References}

1. Giger, M.L., Bae, K.T., MacMahon, H.: Computerized detection of pulmonary nodules in computed tomography images. Investigate. Radiol. 29, 459-465 (1994)

2. Armato, S.G., Giger, M.L., Moran, C.J., Blackburn, J.T., Doi, K., MacMahon, H.: Computerized detection of pulmonary nodules on CT scans. Radiographics 19, 1303-1311 (1999)

3. Kanazawa, K., Kawata, Y., Niki, N., Satoh, H., Ohmatsu, H., Kakinuma, R., Kaneko, M., Moriyama, N., Eguchi, K.: Computer-aided diagnostic system for pulmonary nodules based on helical CT images. In: Doi, K., MacMahon, H., Giger, M.L., Hoffmann, K. (eds.) Computer-Aided Diagnosis Medical Imaging, pp. 131136. Elsevier, Amesterdam, The Netherlands (1999)

4. Lee, Y., Hara, T., Fujita, H., Itoh, S., Ishigaki, T.: Automated detection of pulmonary nodules in helical CT images based on an improved template-matching technique. IEEE Transactions on Medical Imaging 20, 595-604 (2001)

5. Paik, D.S., Beaulieu, C.F., Rubin, G.D., Acar, B., Jeffrey, R.B., Yee Jr., J., Dey, J., Napel, S.: Surface normal overlap: a computer-aided detection algorithm with application to colonic polyps and lung nodules in helical CT. IEEE Transactions on Medical Imaging 23, 661-675 (2004)

6. Zhang, X., McLennan, G., Hoffman, E.A.: Automated detection of small-size pulmonary nodules based on helical CT images. In: Christensen, G.E., Sonka, M. (eds.) IPMI 2005. LNCS, vol. 3565, pp. 664-676. Springer, Heidelberg (2005)

7. Mendoca, P.R.S., Bhotika, R., Sirohey, S.A., Turner, W.D., Miller, J.V.: Modelbased analysis of local shape for lesion detection in CT scans. In: Duncan, J.S., Gerig, G. (eds.) MICCAI 2005. LNCS, vol. 3749, pp. 688-695. Springer, Heidelberg (2005)

8. Ridler, T.W., Calvard, S.: Picture thresholding using an iterative selection method. Man and Cybernetics 8, 630-632 (1978)

9. Besl, P.J., Jain, R.C.: Segmentation through variable-order surface fitting. IEEE Trans. Patt. Anal. Machine Intell. 10, 167-192 (1988)

10. Koenderink, J.J., van Doorn, A.J.: Surface shape and curvature scales. Image and Vision Computing 10, 557-565 (1992)

11. Thirion, J.P., Gourdon, A.: Computing the differential characteristics of isointensity surfaces. Computer Vision and Image Understanding 61, 190-202 (1995) 